\title{
Minocycline as adjunct therapy for a male patient with deficit schizophrenia
}

This article was published in the following Dove Press journal:

Neuropsychiatric Disease and Treatment

\section{Xiaohua Chen ${ }^{1, *}$ \\ Zhenzhen Xiong ${ }^{2, *}$ \\ Zhixiong $\mathrm{Li}^{3}$ \\ Yali Yang ${ }^{4}$ \\ Zhanying Zheng ${ }^{5}$ \\ Yonghong $\mathrm{Li}^{6}$ \\ Yan $\mathrm{Xie}^{6}$ \\ Zhe $\mathrm{Li}^{7,8}$ \\ 'Department of Central}

Transportation Center, West China

Hospital, Sichuan University, Chengdu,

Sichuan 610041, China; ${ }^{2}$ School of

Nursing, Chengdu Medical College,

Chengdu, Sichuan 610083, China;

${ }^{3}$ The Third Department of Clinical

Psychology, Karamay Municipal

People's Hospital, Karamay, Xinjiang

830054, China; ${ }^{4}$ The Second

Department of Clinical Psychology,

Karamay Municipal People's Hospital,

Karamay, Xinjiang 830054, China;

${ }^{5}$ The First Department of Clinical

Psychology, Karamay Municipal

People's Hospital, Karamay, Xinjiang

830054, China; ${ }^{6}$ The Sleep Medicine

Department, Karamay Municipal

People's Hospital, Karamay, Xinjiang

830054, China; ${ }^{7}$ Mental Health Center

and Psychiatric Laboratory, West

China Hospital, Sichuan University,

Chengdu, Sichuan 61004I, China; ${ }^{8}$ The

Mental Rehabilitation Center, Karamay

Municipal People's Hospital, Karamay,

Xinjiang 830054, China

*These authors contributed equally to this work

Correspondence: Zhe Li

The Mental Health Center and Psychiatric Laboratory, West China Hospital,

Sichuan University, No 28 Dian Xin Nan

Road, Chengdu, Sichuan 6I004I, China

Tel +8628 85422633

Fax +86 2885422632

Email jay_li@I63.com

\begin{abstract}
The pathophysiology of schizophrenia may involve increased production of inflammatory cytokines by activated microglia. Minocycline can inhibit activated microglia and may improve secondary negative symptoms and/or cognitive functions when used as adjuvant to antipsychotics. Effects on minocycline on primary and enduring negative symptoms in deficit schizophrenia (DS) are unknown. We present a male patient with a 3-year history of DS. He was treated for 12 weeks with risperidone at a maximal dose of $6 \mathrm{mg}$ per day, then for 10 weeks with olanzapine at $20 \mathrm{mg}$ per day. Symptoms did not improve, and body mass index increased from 20.41 to $22.84 \mathrm{~kg} / \mathrm{m}^{2}$. Serum levels of several inflammatory cytokines were elevated, so we prescribed minocycline as adjunct to aripiprazole for 12 weeks. Negative symptoms and cognitive impairment improved, and serum levels of inflammatory cytokines decreased. Our case suggests that clinicians may consider minocycline as adjunct therapy to antipsychotics in patients with DS with elevated serum levels of inflammatory cytokines. This highlights the need for further research into possible relationships of minocycline with negative symptoms and cognitive function in patients with DS.
\end{abstract}

Keywords: minocycline, adjunct therapy, deficit schizophrenia

\section{Introduction}

Schizophrenia is a devastating mental disorder. Patients with schizophrenia show a variety of symptoms, including hallucination, delusion, blunted affect and emotion, asociality, avolition, cognition impairment, and social withdrawal. Although the etiology of schizophrenia remains unclear, production of various inflammatory cytokines and free radicals by activated microglia may contribute to neuronal degeneration and neurotoxic effects in the disease. ${ }^{1-11}$

Minocycline, a semisynthetic, second-generation tetracycline antibiotic, shows obvious anti-inflammatory and neuroprotective effects that appear to be independent of its antimicrobial action. ${ }^{6,11,12}$ The drug penetrates brain tissue well, is tolerated well, and is nearly completely absorbed when taken orally. ${ }^{13,14}$ It effectively inhibits activated microglia and protects neurons from degeneration in animal models of schizophrenia. ${ }^{15-17}$

Typical and atypical antipsychotics can improve positive symptoms, but show limited efficacy for negative symptoms and cognitive impairments in patients with schizophrenia. ${ }^{2,18}$ Several clinical studies have shown that minocycline can improve negative symptoms and/or cognitive functions of patients with schizophrenia. ${ }^{8,18-22}$

The concept of deficit schizophrenia (DS) has been used to define a subgroup of patients with schizophrenia who show primary and enduring negative symptoms during the clinically stable stage of the disease. The negative symptoms of DS are not 
secondary to anxiety, depression, hallucination, delusion, thought disorder, or medication. Patients who do not meet the criteria of DS are classified as suffering from nondeficit schizophrenia. ${ }^{23,24}$ Their secondary negative symptoms can be treated effectively using second-generation antipsychotics, whereas primary and enduring symptoms often persist despite treatment with conventional or second-generation antipsychotics. ${ }^{24}$ Concentrations of the inflammatory markers IL-6 and C-reactive protein (CRP) are significantly higher in DS patients than in non-DS patients and may be associated with their negative symptoms. ${ }^{25}$ To our knowledge, no study has been reported using minocycline as adjunct therapy to antipsychotics in DS patients.

\section{Case presentation}

Three years before his admission to our hospital, a 26-yearold Chinese man began to manifest, for no apparent reason, restricted affect, poor speech, diminished sense of purpose, and diminished social drive. His affect was diminished no matter what his parents told him. He lacked interest in doing anything, and did not talk to others. When his parents asked him questions, he answered only "I don't know". Sometimes his speech was disorganized and incoherent. He also lost desire to work at his job as a bank clerk, although he previously performed well. He preferred to stay at home and did not want to participate in activities or have contact with friends. His sleep and appetite were poor. He showed no disturbance of consciousness. After these symptoms continued for about 6 months, he was taken by his parents and hospitalized in a local psychiatric ward. General physical and neurologic examinations and routine laboratory investigations at that time showed no notable abnormalities. He was, therefore, diagnosed with schizophrenia and treated with risperidone at a maximal dosage of $6 \mathrm{mg}$ per day for 12 weeks. The patient did not respond, and he was discharged with a weight of $59 \mathrm{~kg}$ and body mass index (BMI) of $20.41 \mathrm{~kg} / \mathrm{m}^{2}$.

After discharge, the patient presented with the same symptoms and he was taken by his parents to our outpatient department 1 year before he was admitted to our hospital. The outpatient physicians diagnosed him with schizophrenia and treated him for 10 weeks with a maximal dose of olanzapine of $20 \mathrm{mg}$ per day. He responded poorly, so he was admitted to our hospital at the request of his parents. The patient reported never smoking and occasionally drinking small amounts of wine. He had no family history of mental disorders. His vital signs were stable, and no abnormal physical or neurologic signs were detected at admission. Blood and urine tests were routine, blood glucose and liver and renal functions were normal, and no evidence of infection was found. His thyroid function was normal. Cerebrospinal fluid (pressure, appearance, cells, proteins, glucose, chloride, and immunoglobulin) and brain magnetic resonance imaging were also normal. The following serum levels were measured: IL- $1 \beta, 8 \mathrm{pg} / \mathrm{mL}$ (reference range, $0-5 \mathrm{pg} / \mathrm{mL}$ ); soluble interleukin-2 receptor (sIL-2R), $812 \mathrm{U} / \mathrm{mL}$ (223-710 U/mL); IL-6, $10 \mathrm{pg} / \mathrm{mL}$ (0-7 pg/mL); IL-8, $51 \mathrm{pg} / \mathrm{mL}(0-62 \mathrm{pg} / \mathrm{mL})$; IL-10, 8.8 $\mathrm{pg} / \mathrm{mL}(0-9.1 \mathrm{pg} / \mathrm{mL})$; tumor necrosis factor- $\alpha(\mathrm{TNF}-\alpha)$, $8.7 \mathrm{pg} / \mathrm{mL}(<8.1 \mathrm{pg} / \mathrm{mL})$; and CRP, $5.5 \mathrm{mg} / \mathrm{L}(<5 \mathrm{mg} / \mathrm{L})$. Electroencephalography, electrocardiography, and transcranial Doppler ultrasound results were normal. The patient and his family members denied any abuse of illicit substances. The patient's weight was $66 \mathrm{~kg}$ and BMI was $22.84 \mathrm{~kg} / \mathrm{m}^{2}$.

The patient was diagnosed with schizophrenia based on the Structured Clinical Interview for the fourth edition of the Diagnostic and Statistical Manual of Mental Disorders, Patient Version. The patient was followed up for 6 months after diagnosis to confirm the presence of schizophrenia. The patient was diagnosed with DS using the Schedule for Deficit Syndrome (SDS). ${ }^{26}$ We dynamically classified the patient and reassessed this classification at 6 and 12 months later using the SDS. The patient scored 82 on the Scale for Assessment of Negative Symptoms (SANS). ${ }^{27}$ Cognitive function was assessed using the Cambridge Neuropsychological Test Automated Battery (CANTAB) (cambridgecognition.com) at admission as well as 10 weeks later (Table 1 ).

Table I Performance on cognitive tests

\begin{tabular}{|c|c|c|c|}
\hline Test & Admission & 12 weeks & Reference values \\
\hline DMS, percent correct ${ }^{\prime}$ & 61 & 89 & 100 (best) \\
\hline $\begin{array}{l}\text { DMS, percent correct } \\
\text { (simultaneous) }\end{array}$ & 79 & 91 & 100 (best) \\
\hline $\begin{array}{l}\text { DMS, percent correct } \\
\text { (all delays)' }\end{array}$ & 50 & 78 & 100 (best) \\
\hline IED, stages completed' & 5 & 5 & 9 (best) \\
\hline $\begin{array}{l}\text { IED, total errors } \\
\text { (adjusted) }^{2}\end{array}$ & 14 & 6 & - \\
\hline $\begin{array}{l}\text { PRM, percent correct, } \\
\text { immediate' }^{\prime}\end{array}$ & 79 & 95 & 100 (best) \\
\hline $\begin{array}{l}\text { PRM, percent correct, } \\
\text { delayed' }\end{array}$ & 59 & 88 & 100 (best) \\
\hline RVP, total false alarms ${ }^{2}$ & 2 & 0 & 0 (best) \\
\hline RVP, probability of hit' & 0.56 & 0.97 & I (best) \\
\hline $\begin{array}{l}\text { SOC, problems solved } \\
\text { in minimum moves' }\end{array}$ & 4 & 9 & 12 (best) \\
\hline SWM, between errors ${ }^{2}$ & 8 & 1 & 0 (best) \\
\hline SWM, strategy ${ }^{2}$ & 32 & 20 & 8 (best)-56 (worst) \\
\hline
\end{tabular}

Notes: 'Higher is better; ${ }^{2}$ Lower is better.

Abbreviations: DMS, delayed matching to sample; IED, intra-/extradimensional shift; PRM, pattern-recognition memory; RVP, rapid visual information processing; SOC, stockings of Cambridge; SWM, spatial working memory. 
The patient was treated with aripiprazole at a maximal dose of $20 \mathrm{mg}$ per day, and treated with minocycline as adjunct therapy, which was gradually titrated to $100 \mathrm{mg}$ twice daily. After 12 weeks of adjunct treatment, persistent and enduring negative symptoms improved. The patient reported no complaints or side effects from the minocycline, he was able to care for himself during daily activities, and his social engagement improved. His communication with parents and contact with friends notably improved. When his parents told him a joke, he laughed. He was willing to exercise and agreed to join a skills training group. The SANS score was 47 , and serum levels of IL- $1 \beta$ were $5 \mathrm{pg} / \mathrm{mL}$; sIL-2R, $670 \mathrm{U} / \mathrm{mL}$; IL-6, 6 pg/mL; IL-8, 48 pg/mL; IL-10, 9.1 pg/mL; TNF- $\alpha$, $7.1 \mathrm{pg} / \mathrm{mL}$; and CRP, $3.6 \mathrm{mg} / \mathrm{L}$. At 12 months follow-up after discharge from our center, he reported no signs of negative symptoms and reported that he was functioning well in the community.

\section{Discussion}

To our knowledge, this is the first successful report of minocycline adjunct treatment for DS. Minocycline effectively reduced primary and enduring negative symptoms in our patient. These antipsychotic effects are likely to be due exclusively to minocycline, because our patient did not suffer any infectious or immune disease.

Our success with minocycline as adjunct therapy to aripiprazole in a patient with DS is consistent with case reports and with randomized, double-blind, placebo-controlled trials indicating that minocycline adjunct therapy can improve negative symptoms in patients with early-phase or chronic schizophrenia. ${ }^{8} 18-20,22,28-30$ These findings are consistent with the notion that during schizophrenia, activated microglia produce various inflammatory cytokines that contribute to neuropathology. ${ }^{6-11}$

Cognitive evaluation of our patient using the CANTAB showed that minocycline led to improvement on several dimensions of attention, memory, and executive function. The Rapid Visual Information Processing Test assesses sustained attention, which is highly sensitive to brain damage or dysfunction. The Pattern-recognition Memory Test assesses visuospatial memory domains. The Delayed Matching to Sample Test assesses simultaneous visual matching ability and short-term visual recognition memory for nonverbalizable patterns. Improvement was also seen on the Battery's three tests of executive function: the Spatial Working Memory Test assesses ability to retain and manipulate information in spatial working memory, the Intra/extradimensional Shift Test assesses cognitive shifting and flexibility, and the Stockings of Cambridge Test assesses planning and organizing a goal-oriented sequence of actions. ${ }^{31}$ Our results suggest that minocycline as adjunct therapy to atypical antipsychotics may decrease damage to different domains of cognitive function, which is consistent with previous studies. ${ }^{19,20}$ In contrast, one study found no significant cognitive difference between patients treated with minocycline or placebo, except in attention. ${ }^{18}$ This discrepancy may reflect the heterogeneity of schizophrenia and differences among cognitive testing tools.

Schizophrenia features diverse symptoms and clinical heterogeneity. These different clinical symptoms may result in different response to antipsychotic treatment. ${ }^{24}$ In our previous work, we found that patients with DS are a relatively homogeneous subgroup of schizophrenia, ${ }^{32-34}$ but the impact of second-generation antipsychotics on DS patients remains controversial. ${ }^{24}$ Several studies have compared the efficacy of amisulpride, clozapine, and olanzapine in treating primary negative symptoms, but without conclusive findings. ${ }^{35-37}$ Our case indicates that minocycline adjunct therapy may be a therapeutic strategy for treating primary and persistent negative symptoms in patients with schizophrenia.

Serum levels of most inflammatory cytokines in our patient decreased after treatment. Conversely, the serum level of IL-10 increased, although it remained within the normal range. Certain atypical antipsychotics may decrease serum levels of cytokines. ${ }^{6,38}$ Meta-analysis of available evidence suggests that IL- $1 \beta$ and IL- 6 are state markers, because they normalize with antipsychotic treatment. In contrast, sIL-2R and TNF- $\alpha$ may be trait markers, because they remain elevated following antipsychotic treatment. ${ }^{4}$ Aripiprazole has been shown to inhibit only levels of inflammatory cytokine TNF- $\alpha,{ }^{39}$ implying that minocycline reduced serum levels of other inflammatory cytokines in our patient, presumably by inhibiting microglial activation.

When treating patients with DS, it may be useful to consider the possibility of neuroinflammation and monitor serum levels of inflammatory cytokines. Large, randomized studies should follow up patients for longer periods after minocycline adjunct therapy in order to assess long-term outcomes and confirm its efficacy against primary and persistent negative symptoms and cognitive function in DS.

\section{Conclusion}

We conclude that minocycline may be considered by clinicians as adjunct therapy in schizophrenia patients with primary and persistent negative symptoms and elevated serum levels of inflammatory cytokines. This highlights the need 
for further research into potential association of minocycline with negative symptoms and cognitive function in patients with DS.

\section{Ethics statement}

Written informed consent for the publication was obtained from the patient and his parents for the case report. This study was approved by the Ethics Committee of West China Hospital, Sichuan University.

\section{Acknowledgments}

The authors would like to thank all of their coworkers of West China Hospital, School of Nursing, Chengdu Medical College and The Mental Rehabilitation Center, Karamay Municipal People's Hospital on this case for their skillful contributions. The authors would like to thank the patient and his parents for their written informed consent to publish this case. This work was partly funded by Science and Technology Department of Sichuan Province of China (2017SZ0049, Zhe Li; 2018ZR0216, Zhenzhen Xiong), Health and Family Planning Commission of Sichuan Province of China (17PJ080, Zhe Li), and The Fundamental Research Funds for The Central Universities of China (2017SCU11072, Zhe Li).

\section{Disclosure}

The authors report no conflicts of interest in this work.

\section{References}

1. Tuominen HJ, Tiihonen J, Wahlbeck K. Glutamatergic drugs for schizophrenia: a systematic review and meta-analysis. Schizophr Res. 2005;72(2-3):225-234.

2. Murphy BP, Chung YC, Park TW, McGorry PD. Pharmacological treatment of primary negative symptoms in schizophrenia: a systematic review. Schizophr Res. 2006;88(1-3):5-25.

3. Chen SL, Lee SY, Chang YH, et al. Inflammation in patients with schizophrenia: the therapeutic benefits of risperidone plus add-on dextromethorphan. J Neuroimmune Pharmacol. 2012;7(3):656-664

4. Miller BJ, Buckley P, Seabolt W, Mellor A, Kirkpatrick B. Meta-analysis of cytokine alterations in schizophrenia: clinical status and antipsychotic effects. Biol Psychiatry. 2011;70(7):663-671.

5. Deng H, Kahlon RS, Mohite S, et al. Elevated plasma S100B, psychotic symptoms, and cognition in schizophrenia. Psychiatr $Q$. 2018;89(1):53-60.

6. Monji A, Kato T, Kanba S. Cytokines and schizophrenia: microglia hypothesis of schizophrenia. Psychiatry Clin Neurosci. 2009;63(3): 257-265.

7. Wehring HJ, Elsobky T, McEvoy JP, et al. Adjunctive minocycline in clozapine-treated patients with schizophrenia: analyzing the effects of minocycline on clozapine plasma levels. Psychiatr Q. 2018;89(1):73-80.

8. Khodaie-Ardakani MR, Mirshafiee O, Farokhnia M, et al. Minocycline add-on to risperidone for treatment of negative symptoms in patients with stable schizophrenia: randomized double-blind placebo-controlled study. Psychiatry Res. 2014;215(3):540-546.

9. Keller WR, Kum LM, Wehring HJ, Koola MM, Buchanan RW, Kelly DL. A review of anti-inflammatory agents for symptoms of schizophrenia. J Psychopharmacol. 2013;27(4):337-342.
10. Monji A. The neuroinflammation hypothesis of psychiatric disorders. Seishin Shinkeigaku Zasshi. 2012;114(2):124-133.

11. Hashimoto K. Microglial activation in schizophrenia and minocycline treatment. Prog Neuropsychopharmacol Biol Psychiatry; 2008;32(7):1758-1759.

12. Filipovic R, Zecevic N. Neuroprotective role of minocycline in cocultures of human fetal neurons and microglia. Exp Neurol. 2008; 211(1):41-51.

13. Kim SS, Kong PJ, Kim BS, Sheen DH, Nam SY, Chun W. Inhibitory action of minocycline on lipopolysaccharide-induced release of nitric oxide and prostaglandin E2 in BV2 microglial cells. Arch Pharm Res. 2004;27(3):314-318

14. Lee SM, Yune TY, Kim SJ, et al. Minocycline inhibits apoptotic cell death via attenuation of TNF-alpha expression following iNOS/NO induction by lipopolysaccharide in neuron/glia co-cultures. $J$ Neurochem. 2004;91(3):568-578.

15. Levkovitz Y, Levi U, Braw Y, Cohen H. Minocycline, a secondgeneration tetracycline, as a neuroprotective agent in an animal model of schizophrenia. Brain Res. 2007;1154:154-162.

16. Zhang L, Shirayama Y, Iyo M, Hashimoto K. Minocycline attenuates hyperlocomotion and prepulse inhibition deficits in mice after administration of the NMDA receptor antagonist dizocilpine. Neuropsychopharmacology. 2007;32(9):2004-2010.

17. Fujita Y, Ishima T, Kunitachi S, et al. Phencyclidine-induced cognitive deficits in mice are improved by subsequent subchronic administration of the antibiotic drug minocycline. Prog Neuropsychopharmacol Biol Psychiatry. 2008;32(2):336-339.

18. Liu F, Guo X, Wu R, et al. Minocycline supplementation for treatment of negative symptoms in early-phase schizophrenia: a double blind, randomized, controlled trial. Schizophr Res. 2014;153(1-3):169-176.

19. Levkovitz Y, Mendlovich S, Riwkes S, et al. A double-blind, randomized study of minocycline for the treatment of negative and cognitive symptoms in early-phase schizophrenia. J Clin Psychiatry. 2010; 71(2):138-149.

20. Chaudhry IB, Hallak J, Husain N, et al. Minocycline benefits negative symptoms in early schizophrenia: a randomised double-blind placebo-controlled clinical trial in patients on standard treatment. J Psychopharmacol. 2012;26(9):1185-1193.

21. Jhamnani K, Shivakumar V, Kalmady S, Rao NP, Venkatasubramanian G. Successful use of add-on minocycline for treatment of persistent negative symptoms in schizophrenia. J Neuropsychiatry Clin Neurosci. 2013;25(1):E06-E07.

22. Miyaoka T, Yasukawa R, Yasuda H, Hayashida M, Inagaki T, Horiguchi J. Possible antipsychotic effects of minocycline in patients with schizophrenia. Prog Neuropsychopharmacol Biol Psychiatry. 2007;31(1):304-307.

23. Carpenter WT Jr, Heinrichs DW, Wagman AM. Deficit and nondeficit forms of schizophrenia: the concept. Am J Psychiatry. 1988; 145(5):578-583.

24. Galderisi S, Maj M. Deficit schizophrenia: an overview of clinical, biological and treatment aspects. Eur Psychiatry. 2009;24(8): 493-500.

25. Garcia-Rizo C, Fernandez-Egea E, Oliveira C, Justicia A, Bernardo M, Kirkpatrick B. Inflammatory markers in antipsychotic-naïve patients with nonaffective psychosis and deficit vs. nondeficit features. Psychiatry Res. 2012;198(2):212-215.

26. Kirkpatrick B, Buchanan RW, McKenney PD, Alphs LD, Carpenter WT Jr. The schedule for the deficit syndrome: an instrument for research in schizophrenia. Psychiatry Res. 1989;30(2):119-123.

27. Andreasen NC. Negative symptoms in schizophrenia. Definition and reliability. Arch Gen Psychiatry. 1982;39(7):784-788.

28. Kelly DL, Vyas G, Richardson CM, et al. Adjunct minocycline to clozapine treated patients with persistent schizophrenia symptoms. Schizophr Res. 2011;133(1-3):257-258.

29. Kelly DL, Sullivan KM, McEvoy JP, et al. Adjunctive minocycline in clozapine-treated schizophrenia patients with persistent symptoms. J Clin Psychopharmacol. 2015;35(4):374-381. 
30. Miyaoka T, Yasukawa R, Yasuda H, Hayashida M, Inagaki T, Horiguchi J. Minocycline as adjunctive therapy for schizophrenia: an open-label study. Clin Neuropharmacol. 2008;31(5):287-292.

31. Levaux MN, Potvin S, Sepehry AA, Sablier J, Mendrek A, Stip E. Computerized assessment of cognition in schizophrenia: promises and pitfalls of CANTAB. Eur Psychiatry. 2007;22(2):104-115.

32. Li Z, Zheng B, Deng W, Liu X, Zheng Z, Li T. Multi-components of evoked-brain potentials in deficit and nondeficit schizophrenia. Asia Pac Psychiatry. 2013;5(2):69-79.

33. Li Z, Deng W, Liu X, et al. Contingent negative variation in patients with deficit schizophrenia or bipolar I disorder with psychotic features: measurement and correlation with clinical characteristics. Nord J Psychiatry. 2015;69(3):196-203.

34. Li Z, Lei W, Deng W, et al. Aberrant spontaneous neural activity and correlation with evoked-brain potentials in first-episode, treatmentnaïve patients with deficit and non-deficit schizophrenia. Psychiatry Res Neuroimaging. 2017;261:9-19.
35. Leucht S, Pitschel-Walz G, Engel RR, Kissling W. Amisulpride, an unusual "atypical" antipsychotic: a meta-analysis of randomized controlled trials. Am J Psychiatry. 2002;159(2):180-190.

36. Rosenheck R, Dunn L, Peszke M, et al. Impact of clozapine on negative symptoms and on the deficit syndrome in refractory schizophrenia. Department of Veterans Affairs Cooperative Study Group on Clozapine in Refractory Schizophrenia. Am J Psychiatry. 1999;156(1):88-93.

37. Lindenmayer JP, Khan A, Iskander A, Abad MT, Parker B. A randomized controlled trial of olanzapine versus haloperidol in the treatment of primary negative symptoms and neurocognitive deficits in schizophrenia. J Clin Psychiatry. 2007;68(3):368-379.

38. GirgisRR,DiwadkarVA,NutcheJJ, SweeneyJA,Keshavan MS, HardanAY. Risperidone in first-episode psychosis: a longitudinal, exploratory voxel-based morphometric study. Schizophr Res. 2006;82(1):89-94.

39. Kato T, Mizoguchi Y, Monji A, et al. Inhibitory effects of aripiprazole on interferon-gamma-induced microglial activation via intracellular Ca2+ regulation in vitro. J Neurochem. 2008;106(2):815-825.
Neuropsychiatric Disease and Treatment

\section{Publish your work in this journal}

Neuropsychiatric Disease and Treatment is an international, peerreviewed journal of clinical therapeutics and pharmacology focusing on concise rapid reporting of clinical or pre-clinical studies on a range of neuropsychiatric and neurological disorders. This journal is indexed on PubMed Central, the 'PsycINFO' database and CAS,

\section{Dovepress}

and is the official journal of The International Neuropsychiatric Association (INA). The manuscript management system is completely online and includes a very quick and fair peer-review system, which is all easy to use. Visit http://www.dovepress.com/testimonials.php to read real quotes from published authors.

Submit your manuscript here: http://www.dovepress.com/neuropsychiatric-disease-and-treatment-journal 